\title{
Electron Energy-Loss Spectroscopy of Organic Photovoltaics.
}

Frank J. Scheltens ${ }^{1}$, Lawrence F. Drummy ${ }^{2}$, Michael F. Durstock ${ }^{2}$, James B. Gilchrist ${ }^{3}$, Sandrine Heutz ${ }^{3}$ Jessica A. Alexander ${ }^{1}$, and David W. McComb ${ }^{1}$

${ }^{1 .}$ Center for Electron Microscopy and Analysis, Department of Materials Science and Engineering, The Ohio State University, Kinnear Road, Columbus OH 43212, United States

2. Materials and Manufacturing Directorate, Air Force Research Laboratory, WPAFB, Ohio 45433, United States

3. Department of Materials, Imperial College London, SW7 2AZ, United Kingdom

Advances in organic photovoltaic (OPV) based solar cell device technology have increased power conversion efficiencies (PCE) beyond 11\% [1], pushing flexible architecture OPV devices closer to being a viable low-cost, environmentally friendly alternative to contemporary inorganic based solar cells $[2,3]$. Extending OPV performance beyond this limit is a critical challenge that requires better understanding of the PCE limiting processes. Since only photo-generated excitons that diffuse to the interface between electron donor and acceptor materials can dissociate into holes and electrons, understanding the chemistry and molecular structure of this interface is critical to identifying and mitigating these limitations. Other factors such as the amount of light absorption, efficiency of photogeneration of electrons and holes, and their collection efficiency at the respective electrodes must also be optimized in order to improve the device PCE. Electron energy-loss spectroscopy (EELS) is an extremely useful tool that can be used to probe the nature and structure of these interfaces and further the understanding of processes that occur there.

In this contribution we describe results from $30 \mathrm{~nm}$ thick films of copper phthalocyanine (CuPc), templated by a $5 \mathrm{~nm}$ layer of 3,4,9,10-perylene tetracarboxylic dianhydride (PTCDA) grown on potassium chloride (KCL) substrates by evaporation in a Kurt J. Lesker high vacuum chamber with a base pressure about $10^{-8}$ mbar and a growth rate of $1 \mathrm{As}^{-1}$. The CuPc films were floated off the $\mathrm{KCl}$ substrates with deionized water and collected onto lacey carbon coated grids. Monochromated low loss EELS was performed on these films using an FEI Titan 60-300 transmission electron microscope operating in scanning transmission electron microscopy (STEM) mode at $60 \mathrm{kV}$. EELS data was collected using a Gatan GIF Model 966 Quantum ERS imaging filter and then processed and analyzed using the Kramers-Kronig implementation within Gatan Digital Micrograph software.

A monochromated low loss EELS spectrum collected from a plan view $\mathrm{CuPc}$ thin film was processed to remove the zero loss peak (ZLP) and then corrected for plural scattering. The resulting single scattering distribution, $\mathrm{S}(\mathrm{E})$, shown in Figure 1a, displays a number of features between the energy loss values of $1 \mathrm{eV}$ to $10 \mathrm{eV}$, corresponding to the optical range of the solar spectrum. The extraction of the $\mathrm{S}(\mathrm{E})$ from the collected valence loss data is not a trivial process. Substantial issues exist that are related to the proper removal of the monochromated ZLP from the low loss EELS spectra. After performing a Kramers-Kronig analysis of this $\mathrm{S}(\mathrm{E})$, the resulting imaginary part of the complex dielectric function, shown in Figure 1b, reveals single electron transitions and collective excitations of the CuPc in the same solar energy range. This nano-localized EELS based imaginary dielectric function measurement correlates well with bulk light optical based ellipsometry measurements shown in Figure 1c. 


\section{References:}

[1] Chen, C.-C. et al., Advanced Materials 26 (2014), 5670.

[2] C.J. Brabec, N.S. Sariciftci, et al., Advanced Functional Materials 11 (2001), 15.

[3] N.S. Lewis, Science 315 (2007), 798.
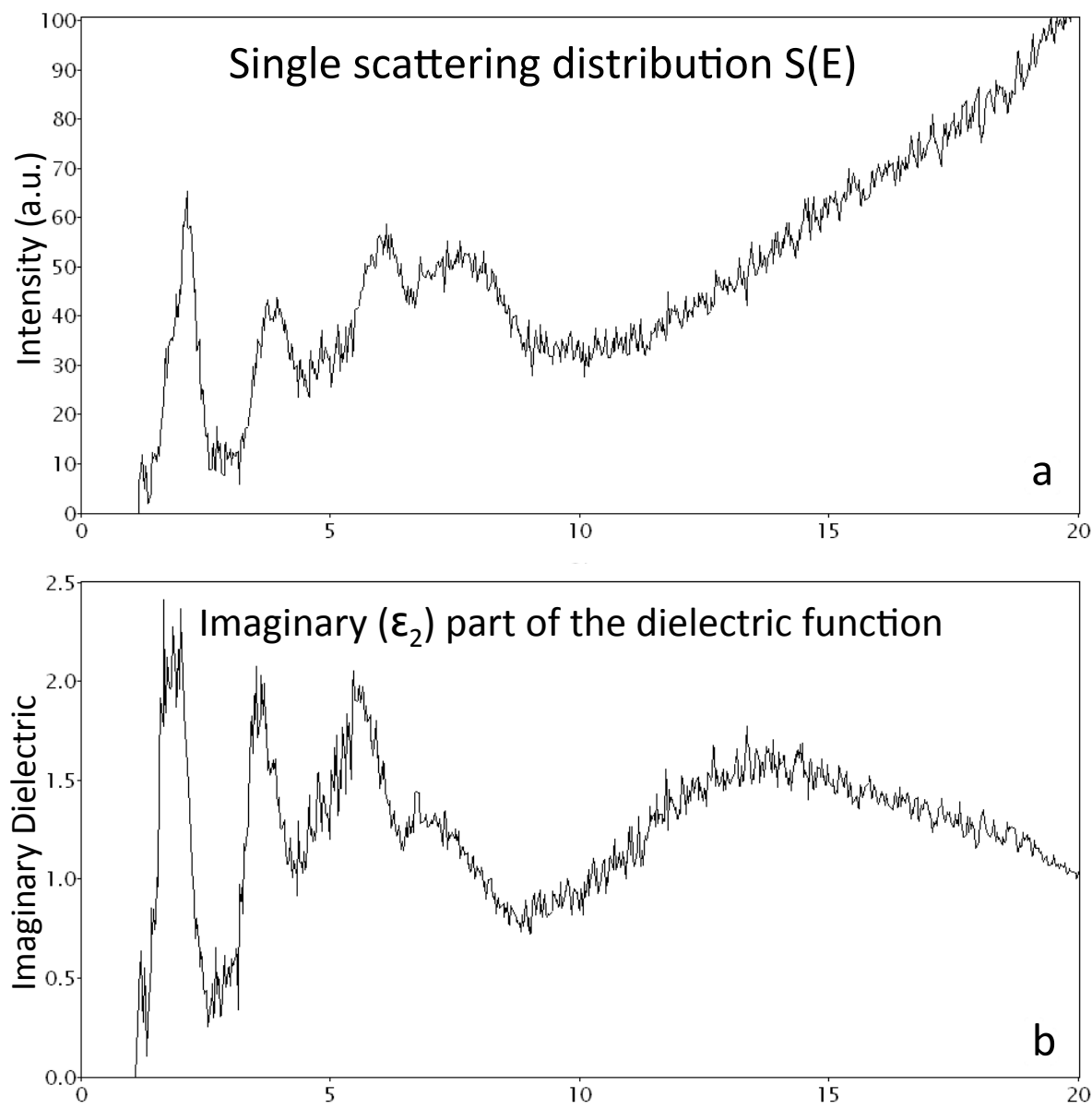

-

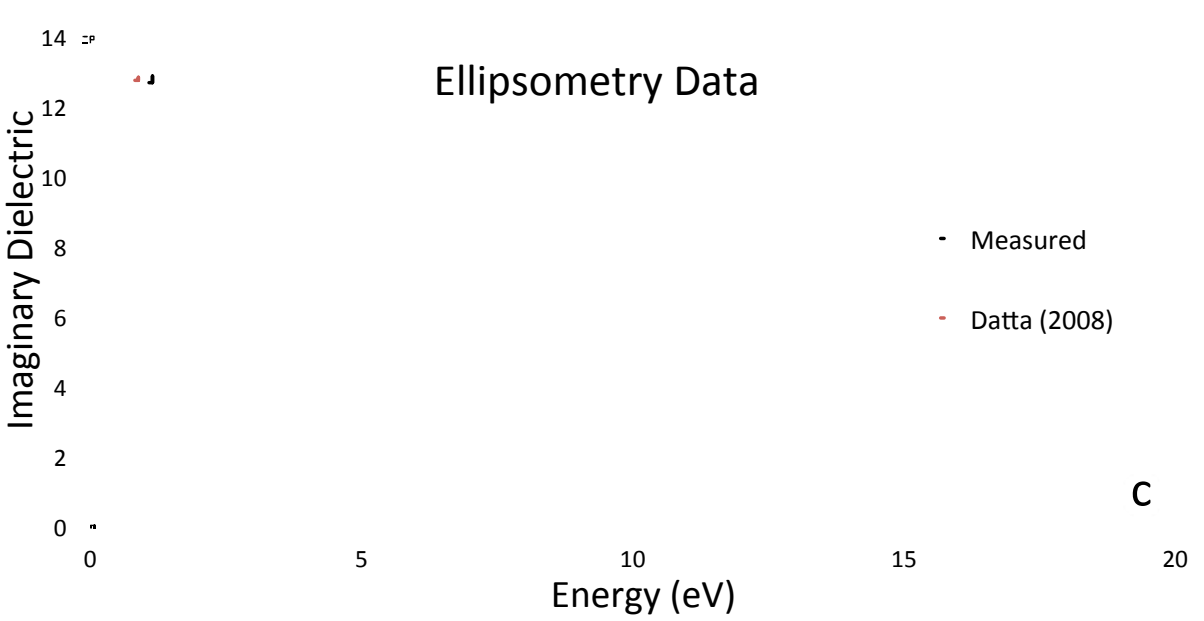

Figure 1. Low loss EELS of evaporated CuPc film: a) Single scattering distribution $\mathrm{S}(\mathrm{E})$; b) Imaginary $\left(\varepsilon_{2}\right)$ part of the dielectric function calculated from $\left.\mathrm{S}(\mathrm{E}) ; \mathrm{c}\right)$ Imaginary $\left(\varepsilon_{2}\right)$ part of the dielectric function from ellipsometry. 